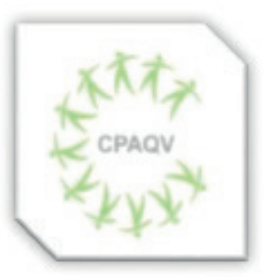

ISSN: 2178-7514

Vol. $12\left|\mathbf{N}^{\circ} .3\right|$ Ano 2020
ARTIGO ORIGINAL

\section{OS EFEITOS DA CARBOXITERAPIA NAS ATROFIAS CUTÂNEAS ALBAS LOCALIZADAS NA REGIAO GLÚTEA DE MULHERES}

The effects of carboxytherapy on the albas cutaneous atrophies located in the gluty region of women

Tereza Cristina dos Reis Ferreira ${ }^{1}$, Amanda de Souza², Patrícia Damasceno da Silva², Amanda Jordana Silva Souza ${ }^{3}$, Luane Vanzeler Monteiro ${ }^{4}$ Júlio César Veiga Pena ${ }^{4}$, Paula Thayna Soares Lima ${ }^{4}$, Paulo Vitor de Souza Sassim4, Antônio Gabriel Pantoja Silva Santos ${ }^{4}$, Gleidiane Lorrana Sales dos Santos ${ }^{4}$, Raquel de Souza Mota ${ }^{4}$, Ana Eveni Rodrigues Aguiar ${ }^{4}$, Gabriel Vinícius Reis de Queiroz ${ }^{5}$

\title{
RESUMO
}

Estria é uma atrofia tegumentar adquirida que surge quando as fibras elásticas e colágenas (responsáveis pela firmeza da pele) se rompem e formam "cicatrizes". As estrias ocorrem mais em mulheres, podendo ser discretas ou exuberantes.quando há destruição de fibras elásticas e colágenas, que são responsáveis pela sustentação e elasticidade da pele. As estrias podem não ser causadas por doenças subjacentes. Algumas causas comuns são gravidez, ganho de peso na obesidade, estirões de crescimento em adolescentes, efeitos colaterais de medicamentos ou rápido aumento do músculo devido a musculação. A terapia de dióxido de carbono (CO2) refere-se à administração transcutânea e subcutânea de $\mathrm{CO} 2$ para fins terapêuticos. Caracteriza-se pelo uso terapêutico do gás carbônico medicinal com 99,9\% de pureza administrado de forma subcutânea (percutânea) tendo como objetivo uma vasodilatação periférica e melhora da oxigenação tecidual, $\mathrm{O}$ objetivo deste estudo foi verificar os efeitos da carboxiterapia nas estrias albas localizadas na região glútea em mulheres jovens. Foram realizadas 10 aplicações de carboxiterapia em ambos os glúteos de cada paciente, sendo feitas avaliações e reavaliações nas pacientes para coleta de dados, sendo adotado um nível de confiança de 5\% e 7 graus de liberdade, para este caso o valor tabelado foi t7;0,05 =1,90, para o teste unicaudal. Os resultados deste estudo, sugerem que a carboxiterapia é um recurso que trouxe resultados estatisticamente significantes a respeito da satisfação corporal das pacientes, colaborando com aspecto social e psicológico das mesmas, assim como uma melhor auto-estima com o decorrer das aplicações.

Palavras-chave: Estrias, cicatrização, Fisioterapia

\section{ABSTRACT}

Stria is an acquired cutaneous atrophy that arises when the elastic and collagen fibers (responsible for the firmness of the skin) break and form "scars". Stretch marks occur more in women, and can be discreet or exuberant.when there is destruction of elastic and collagen fibers, which are responsible for the support and elasticity of the skin. Stretch marks may not be caused by underlying diseases. Some common causes are pregnancy, weight gain in obesity, growth strains in adolescents, side effects of medications or rapid muscle growth due to weight training. Carbon dioxide (CO2) therapy refers to the transcutaneous and subcutaneous administration of $\mathrm{CO} 2$ for therapeutic purposes. It is characterized by the therapeutic use of medicinal carbon dioxide with $99.9 \%$ purity administered subcutaneously (percutaneously) with the objective of peripheral vasodilation and improvement of tissue oxygenation. in the gluteal region in young women. 10 carboxytherapy applications were carried out in both buttocks of each patient, with evaluations and reevaluations in the patients for data collection, with a confidence level of $5 \%$ and 7 degrees of freedom, for this case the tabulated value was $\mathrm{t} 7 ; 0.05=1.90$, for the one-tailed test. The results of this study suggest that carboxitherapy is a resource that brought statistically significant results regarding the patients' body satisfaction, collaborating with their social and psychological aspects, as well as a better self-esteem over the course of the applications.

Keywords: Atrophy, Wound Healing, Physical Therapy Specialty

1- Fisioterapeuta, Dra em Ciências da Reabilitação (UNINOVE)

2- Fisioterapeuta, Graduada pelo Centro Universitário do Estado do Pará (CESUPA)

3- Fisioterapeuta, Pós-graduação Latu Senso em Saúde da Familia (CESUPA)

4- Discente do Curso de Fisioterapia da Universidade do Estado do Pará (UEPA)

5- Fisioterapeuta, Graduado pela Universidade da Amazônia (UNAMA)

Autor de correspondência

Tereza Cristina dos Reis Ferreira

tereza.reis@uepa.br

DOI: $\underline{10.36692 / v 12 n 3-49}$ 


\section{INTRODUÇÃO}

O tegumento recobre toda a superfície do corpo e apresenta-se constituído por duas camadas: a epiderme e a derme ${ }^{[4,8,10]}$. Essas camadas estão propensas a sofrer alguns danos. Sendo assim, quando ocorre um rompimento das fibras elásticas da pele, por um comprometimento do colágeno, por um estresse mecânico (estiramento), estresse fisiológico (estímulo hormonal) ou por predisposição genética, formam-se as estrias ${ }^{[1,6,11]}$.

Os métodos utilizados no tratamento das estrias visam melhorar os aspectos estéticos, estimulando a formação de tecido colágeno nas lesões. Para isso existem várias técnicas, dentre estas técnicas destaca-se a carboxiterapia ${ }^{[3,5,7]}$.

A terapia de dióxido de carbono (CO2) refere-se à administração transcutânea e subcutânea de CO2 para fins terapêuticos. Caracteriza-se pelo uso terapêutico do gás carbônico medicinal com 99,9\% de pureza administrado de forma subcutânea (percutânea) tendo como objetivo uma vasodilatação periférica e melhora da oxigenação tecidual ${ }^{[2,9]}$.

Este estudo teve como objetivo geral verificar os efeitos da carboxiterapia nas estrias albas em glúteos de mulheres jovens, analisando o comprimento e a largura das estrias, o nível de sensibilidade nas estrias por meio dos monofilamentos Semmes-Weinstein, o nível de dor por meio da escala análoga de dor e o nível de satisfação das pacientes durante a avaliação e a reavaliação.

\section{MÉTODOS}

Este estudo foi submetido e aprovado no CEP do Centro Universitário do Pará sob número 0051.0.323.000-11. O estudo em questão é do tipo experimental, quantitativo e prospectivo. A amostra foi constituída por oito mulheres, com estrias albas na região glútea.

Foram incluídas no estudo mulheres com idade entre 20 e 30 anos, sem histórico de gestações, e com tipo de pele entre III e IV de acordo com a classificação de Fitzpatrick. Foram excluídas mulheres com histórico de gestações, gestantes, mulheres com idade inferior a 20 anos e superior a 30 anos, mulheres que possuíam o tipo de pele I, II, V e VI de acordo coma classificação de Fitzpatrick, mulheres que usavam anticoncepcionais e mulheres que apresentavam alguma contra indicações para carboxiterapia.

A coleta de dados foi realizada de acordo com o protocolo da Ficha de Avaliação das Atrofias Lineares Cutâneas, segundo Guirro e Guirro $^{[6]}$, sendo que a mesma foi modificada para que houvesse um maior direcionamento da avaliação para as estrias albas.

Foram realizadas 10 aplicações de carboxiterapia em ambos os glúteos de cada paciente, sendo feitas avaliações e reavaliações nas pacientes para coleta de dados, sendo adotado um nível de confiança de 5\% e 7 graus de liberdade, para este caso o valor tabelado foi t7;0,05 =1,90, para o teste unicaudal. 


\section{RESULTADOS E DISCUSSÃO}

Em relação à paquimetria do comprimento das estrias no glúteo direito antes e depois do tratamento, observa-se que a diferença entre as medidas das estrias é de -26,85 mm em média com Desvio Padrão de 12,85, onde se adotou o nível de confiança de 5\% e $(\mathrm{gl}=\mathrm{n}-1) 7$ graus de liberdade, neste caso com o valor tabelado de t7;0,05 =1,90, para o teste unicaudal, mostrando significância estatística com tc de -5,53.

Em relação à paquimetria da largura das estrias no glúteo direito antes e depois do tratamento, observa-se que a diferença entre as medidas das estrias é de -1,19 mm em média, com Desvio Padrão de 1,07, onde se adotou o nível de confiança de 5\% e (gl=n-1) 7 graus de liberdade, neste caso com o valor tabelado de t7;0,05 =1,90, para o teste unicaudal, mostrando significância estatística com tc de -2,94. Analisando a paquimetria do comprimento das estrias do glúteo esquerdo antes e depois do tratamento, observa-se que a diferença entre as medidas das estrias é de $-32,25 \mathrm{~mm}$ em média com Desvio Padrão de 12,37, onde se adotou o nível de confiança de $5 \%$ e $(\mathrm{gl}=\mathrm{n}-1) 7$ graus de liberdade, neste caso com o valor tabelado de t7;0,05 =1,90, para o teste unicaudal, mostrando significância estatística com tc de -6,90.

Analisando a paquimetria da largura das estrias do glúteo esquerdo, foi possível observar que a média antes e depois do tratamento é de -1,44 mm, com Desvio Padrão de 0,86, onde se adotou o nível de confiança de $5 \%$ e $(\mathrm{gl}=\mathrm{n}$ 1) 7 graus de liberdade, neste caso com o valor tabelado de t7;0,05 =1,90, para o teste unicaudal, mostrando significância estatística com tc de $-4,40$.

Verifica-se que para todos os locais onde o procedimento foi aplicado houve diferença significativa. Pode-se concluir para este caso que houve diminuição das estritas, com acentuação maior para o lado esquerdo tanto para o comprimento quanto para a largura.

\begin{tabular}{|c|c|c|c|c|c|}
\hline $\begin{array}{c}\text { LOCAL DO } \\
\text { PROCEDIMENTO }\end{array}$ & $\begin{array}{l}\text { MÉDIA } \\
\text { INICIAL }\end{array}$ & $\begin{array}{l}\text { MEDIA } \\
\text { FINAL }\end{array}$ & $\begin{array}{c}\text { DIFERENÇA } \\
\text { MÉDIA }\end{array}$ & $\begin{array}{l}\text { DESVIO- } \\
\text { PADRÃO }\end{array}$ & TC \\
\hline \multicolumn{6}{|l|}{ Comprimento } \\
\hline Direito & 52 & 25,12 & $-26,88$ & 12,86 & $-5,53^{* * *}$ \\
\hline Esquerdo & 55,62 & 23,37 & $-32,25$ & 12,37 & $-6,90^{\star * * *}$ \\
\hline $\begin{array}{l}\text { Largura } \\
\text { Direito }\end{array}$ & 1,75 & 0,56 & $-1,19$ & 1,07 & $-2,94^{* *}$ \\
\hline Esquerdo & 1,87 & 0,43 & $-1,44$ & 0,86 & $-4,40^{* * *}$ \\
\hline
\end{tabular}

Fonte: Pesquisa de Campo, 2011.

** Significativo ao nível de 0,01 ; *** Significativo ao nível de 0,005 ; *** Sianificativo ao nível de 0.001 
Brandi et $\mathrm{al}^{[2]}$, através de um estudo histológico com a Carboxiterapia, intitulado Carbon Dioxide therapy in the treatment of localized adiposities: clinical study and histopathological correlations, comprovou um aumento da espessura da derme, evidenciando estímulo à neocolagenase, bem como preservação total do

tecido conjuntivo, incluindo estruturas vasculares e nervosas, ou seja, um evidente rearranjo das fibras colágenas.

Domingues e Macedo $^{[5]}$, através de sua pesquisa intitulada Efeito microscópico do dióxido de carbono na atrofia linear cutânea, onde foi feita análise histológica em 10 mulheres com estrias brancas nacaradas antes a após as aplicações de carboxiterapia, comprovaram que a aplicação de CO2 para o tratamento das estrias apresentou grande significância quanto a alteração do aspecto da pele, tanto na epiderme quanto na derme, pois inicialmente, todos os sujeitos da amostra apresentavam derme com fibrose dérmica e ao final do tratamento passaram a apresentar aspecto de feixes conjuntivos regulares.

Após as 10 sessões, este estudo sugere que algum efeito fisiológico da terapia por dióxido de carbono apresentou tendência de melhora quanto ao aspecto macroscópico em relação ao comprimento e largura das estrias.

A respeito da sensibilidade dolorosa quanto à aplicação de gás no local das estrias, durante a primeira e a última sessão, verificou- se que $62,5 \%$ das pacientes relataram uma diminuição da sensibilidade dolorosa e 37,5\% relataram um aumento da sensibilidade dolorosa.

Domingues e Macedo ${ }^{[5]}$ pode observar a diminuição da sensação de dor percebida pelas pacientes ao longo das sessões de tratamento podendo confirmar a evolução positiva, por meio da redução da dor ao longo do tratamento. No presente estudo foi aplicada a escala visual análoga de dor para verificar a sensibilidade dolorosa quanto à aplicação de gás no local das estrias, durante a primeira e a última sessão, onde verificou-se que $62,5 \%$ das pacientes relataram uma diminuição da sensibilidade dolorosa, ou seja, ouve uma acomodação da sensação de dor percebida pela maioria das pacientes.

Em relação à satisfação das pacientes, através de uma escala de zero a dez, onde quanto mais próximo do zero, menor a satisfação pessoal e quanto mais próximo de dez, maior a satisfação pessoal, pudemos notar, que a média inicial de satisfação foi de 6,25 e a média final da satisfação foi de 9,25, ou seja, as pacientes ficaram mais satisfeitas com o seu corpo após as aplicações de carboxiterapia.

Devido à escassa literatura, não foram encontrados relatos fidedignos em outros estudos a respeito da satisfação corporal das pacientes antes e/ou após a aplicação de carboxiterapia nas estrias. Neste sentido, o presente trabalho avaliou a satisfação corporal das pacientes antes e após todos os atendimentos de carboxiterapia, através de uma escala de zero a dez, onde quanto 
mais próximo de dez, maior é a satisfação. Assim, pudemos notar, que a média inicial de satisfação foi de 6,25 e a média final da satisfação foi de 9,25, ou seja, todas as pacientes indicaram maior satisfação corporal, em relação às estrias, após as aplicações desta técnica.

Por meio dos monofilamentos de Semmes-Weinstein, o qual adota valores a cada monofilamentos indicando que quanto menor o resultado do score significa menor a alteração da sensibilidade do indivíduo e vice-versa, 100\% da amostra apresentou, no momento da avaliação e reavaliação, sensibilidade para o monofilamento verde, cujo valor é zero, caracterizando uma boa sensibilidade, por parte das pacientes, no local acometido pelas estrias.

Segundo Guirro e Guirro ${ }^{[6]}$,em estrias profundas a sensibilidade está alterada e a regeneração da mesma propicia o retorno de todas as funções inerentes à pele, inclusive a sensitiva, que se encontrava grandemente diminuída.

\section{CONCLUSÃO}

Os resultados deste estudo, sugerem que a carboxiterapia é um recurso que trouxe resultados estatisticamente significantes a respeito da satisfação corporal das pacientes, colaborando com aspecto social e psicológico das mesmas, assim como uma melhor auto-estima com o decorrer das aplicações.

\section{REFERÊNCIAS}

1. AZULAY, D. R.; AZULAY, M. M. Estrias. In: KEDE, M. P. V.; SABATOVICH,

O. Dermatologia Estética. São Paulo: Atheneu, 2004. $363-368$ p.

2. BRANDI, C. et al. Carbon dioxide therapy in the treatment of localized adiposities: clinical study and histopathological correlations. Aesthetic Plastic Surgery, Italy, n. 25, p. 170-174, 2001. Disponível em: http:// www.chirurgie-dermatologique.com/ upload/forum/ Image/1234.pdf. Acesso em: 21 out. 2011.

3. BORGES, F. S. Dermatofuncional: Modalidadesterapêuticas nas disfunções estéticas 2 ed. São Paulo: Phorte, 2010.

4. CORMACK, D.H. Fundamentos de histologia. 2 ed. Rio de Janeiro: Guanabara Koogan, 2003. P.229237.

5. DOMingues, A. C. S; MACEDO, C. S. A. C. Efeito microscópio do dióxido de carbono na atrofia linear cutânea. 2006. 53f. Trabalho de Conclusão de

Curso (Bacharel em Fisioterapia) - Universidade da Amazônia, Belém, 20006 . Disponível em: http://www.unama.br:8080/graduacao/ cursos/Fisioterapia/tcc/2006/efeito- microscopiodo-dioxido-de-carbono-na-atrofia-linear-cutanea.pdf. Acesso em: 05 mar. 2011.

6. GUIRRO, R. R.; GUIRRO, E. C. O. Fisioterapia dermato-funcional: Fundamentos, Recursos e Patologias 3.ed. Barueri: Manole, 2004. p. 391- 412

7. IBRAMED. Instruções de uso: carboxiterapia ares. São Paulo, Ed. 1, dez. 2009.Disponível em: http:/ / www.ibramed.com.br/upload/produtos_74_manual_ pt.pdf. Acessado em: 16 out. 2011.

8. JUNQUEIRA, L. C.; CARNEIRO, J. Histologia básica. 8. Ed. Rio de Janeiro: Guanabara Koogan, 1995.

9. LEE, G.S.K. Carbon Dioxide Therapy in the Treatment of Cellulite: An Audit of Clinical Practice. Aesthetic Plastic Surgery, n. 34, p. 239-243, jan. 2010. Disponivel em: http://www.ncbi.nlm.nih.gov/pmc/ articles/PMC2847160/?tool=pubmed. Acessado em: 14 nov. 2011.

10. OVALLE, W. K. ; NETTER, F.H.; NAHIRNEY, P. C. Netter bases da

histologia. Rio de Janeiro: Elsevier, 2008. p. 245-253.

11. SÊNOS E. M.; CASTELLO BRANCO, K. S. G. Estrias. In: RAMOS E

SILVA, M.; CASTRO, M.C.R. Fundamentos de dermatologia, edição revisada e atualizada. 2. ed. Rio de Janeiro: Atheneu, 2010. 1959-1966p.

OBSERVAÇÃO: Os autores declaram não existir conflitos de interesse de qualquer natureza. 